\title{
Application of a Combined Model for Spare Parts Consumption Prediction
}

\author{
Liu Shenyang*, Gao Qi, Gao Tielu, Ge Yang and Li Zhiwei \\ Department of Equipment Command \& Management of Mechanical Engineering College, Shijiazhuang, Hebei, 050003, \\ P.R. China
}

\begin{abstract}
Spare parts consumption prediction lays a foundation for spare parts support. This paper combined the exponential smoothing method with a grey model to establish a combined model. The combined model solved the problem of spare parts consumption prediction. The example indicated that the combined model is much more accurate than a single model.
\end{abstract}

Keywords: Combined model, Exponential smoothing method, Grey model, Spare parts consumption.

\section{INTRODUCTION}

The spare parts consumption includes the amount of spare parts used to maintain the specific amount of equipments at the specific states under specific times and conditions. Nearly all the segments about spare parts include acquisition, storage with supply and management having close connections with the analysis of information regarding spare parts consumption. We can project reasonable spare parts support scheme and enhance the scientific lever of spare parts support work only if we master the law of spare parts consumption.

Many scholars have made scientific researches on spare parts consumption prediction. Zhao Jianzhong improved the search mode of APSO and weighted method of least squares support vector machine. Following this, the consumption forecasting model of missile spare parts was established based on RS, EW and WLS-SVM with APSO, and the realization process was analyzed. The results showed that the combinatorial forecasting model has better forecast precision and important applied value in the course of forecasting the consumption of missile spare parts [1]. Ni Xiancun by using the concept of the repair degree, improved the proportional hazards model based on general renewal process. The parameter value was estimated by analyzing failure data and then the number of tables was calculated based on Monte Carlo simulation. An example was given and the results of various maintenance policies with and without considering covariates were compared and analyzed. Results showed that the model had a larger practical value [2]. Li Dawei used the initial spare parts scheme as prior information and proposed to regulate the method of spare parts in the incipient operation based on the Bayes method. Finally, the simulation example showed that the proposed method was feasible. Compared with the classical method, the proposed method

*Address correspondence to this author at Department of Equipment Command \& Management of Mechanical Engineering College, Shijiazhuang, Hebei, 050003, P.R. China; Tel: 15127134769;

E-mail: 1037279749@qq.com can improve the accuracy of the estimation of spare parts consumption with steadiness. In this way, a more rational spare parts scheme can be formed [3].

In the practice of predicting spare parts consumption, there are not enough historical data to be used to forecast due to a variety of reasons, and the small sample data of spare parts consumption are the only available information. The traditional forecasting methods provide inaccurate results on small sample data. Therefore, a combined model for spare parts consumption prediction based on exponential smoothing method and grey model was developed to avoid the limitations of single prediction method, utilize all the information, and improve the prediction of spare parts consumption. This combined model works well with small sample data.

\section{MODELING}

\subsection{Exponential Smoothing Method}

The exponential smoothing method has some specific models such as the single exponential smoothing model, the double exponential smoothing model and the cubic exponential smoothing model $[4,5]$. The single exponential smoothing model could be used when the time series is stable, and the trend shows a horizontal direction. The double exponential smoothing model could be used when the time series shows a linear direction. The cubic exponential smoothing model could be used when the time series shows a non-linear direction.

(1) The formula of single exponential smoothing is $S_{t}^{(1)}=\alpha x_{t}+(1-\alpha) S_{t-1}^{(1)}$ and the prediction model of single exponential smoothing is $\hat{x}_{t+1}=S_{t}^{(1)}=\alpha x_{t}+(1-\alpha) \hat{x}_{t}$.

(2) The formula of double exponential smoothing is $S_{t}^{(2)}=\alpha S_{t}^{(1)}+(1-\alpha) S_{t-1}^{(2)}$ and the prediction model of double exponential smoothing is $\hat{x}_{t+T}=a_{t}+b_{t} T$, where 
Table 1. Accuracy indicators of the grey model.

\begin{tabular}{|c|c|c|c|}
\hline \multirow{2}{*}{ Grade } & Relative Error & Ratio of Standard Deviation & Small Error Probability \\
& $\Delta$ & 0 & 0.35 \\
\hline \hline One & 0.01 & 0.50 & 0.90 \\
\hline Two & 0.05 & 0.65 & 0.80 \\
\hline Three & 0.10 & 0.80 & 0.70 \\
\hline Four & 0.20 & 0.60 \\
\hline
\end{tabular}

$a_{t}=2 S_{t}^{(1)}-S_{t}^{(2)}, b_{t}=\frac{\alpha}{1-\alpha}\left(S_{t}^{(1)}-S_{t}^{(2)}\right)$

(3) The formula of cubic exponential smoothing is $S_{t}^{(3)}=\alpha S_{t}^{(2)}+(1-\alpha) S_{t-1}^{(3)}$ and the prediction model of cubic exponential smoothing is $\hat{x}_{t+T}=a_{t}+b_{t} T+\frac{1}{2} c_{t} T^{2}$, where

$$
\begin{aligned}
& a_{t}=3 S_{t}^{(1)}-3 S_{t}^{(2)}+S_{t}^{(3)} \\
& b_{t}=\frac{\alpha}{2(1-\alpha)^{2}}\left[(6-5 \alpha) S_{t}^{(1)}-2(5-4 \alpha) S_{t}^{(2)}+(4-3 \alpha) S_{t}^{(3)}\right] \\
& c_{t}=\frac{\alpha^{2}}{(1-\alpha)^{2}}\left[S_{t}^{(1)}-2 S_{t}^{(2)}+S_{t}^{(3)}\right] . \\
& S_{t}^{(1)}, S_{t}^{(2)} \text { and } S_{t}^{(3)} \text { refer to the } t^{t h} 1-3 \text { order exponential }
\end{aligned}
$$
smoothing predictive value, and $S_{t-1}^{(1)}, S_{t-1}^{(2)}$ and $S_{t-1}^{(3)}$ refer to the $(t-1)^{t h} 1-3$ order exponential smoothing predictive value, $x_{t}$ and $\ddot{x}_{t}$ are the $t^{t h}$ actual value and estimated value, respectively, $T$ is the number of time points from the initial to the target time point, and $\alpha$ is a smoothing coefficient

$(0<\alpha<1)$.

\subsection{Grey Model}

The scope of application of a grey model depends on the situation when the changes in the spare parts consumption data are close to the exponential function. The time series based on the spare parts consumption data collected is shown as:

$$
x^{(0)}=\left\{x^{(0)}(1), x^{(0)}(2), \mathrm{L}, x^{(0)}(n)\right\} \quad x^{(0)}(i) \geq 0, \quad i=1,2, \mathrm{~L}, n .
$$

After accumulative operations, the new time series becomes :

$$
x^{(1)}=\left\{x^{(1)}(1), x^{(1)}(2), \mathrm{L}, x^{(1)}(n)\right\}
$$

Where $x^{(1)}(k)=\sum_{i=1}^{k} x^{(0)}(i), k=1,2, \mathrm{~L}, n$.

The mean sequence of $x^{(1)}$ is noted as $z^{(1)}$ [6], which is $z^{(1)}(k)=0.5 x^{(1)}(k)+0.5 x^{(1)}(k-1)$

$z^{(1)}=\left\{z^{(1)}(2), z^{(1)}(3), \mathrm{L}, z^{(1)}(n)\right\}$.

The grey model is

$$
x^{(0)}(k)+a z^{(1)}(k)=b,
$$

Where $a$ and $b$ are grey coefficients.

The estimated values of $a$ and $b$ are acquired by the least square method. Because of

$$
\begin{aligned}
& B=\left[\begin{array}{cccc}
-z^{(1)}(2) & -z^{(1)}(3) & \mathrm{L} & -z^{(1)}(n) \\
1 & 1 & \mathrm{~L} & 1
\end{array}\right]^{T}, \\
& y_{N}=\left[x^{(0)}(2), x^{(0)}(3), \mathrm{L}, x^{(0)}(n)\right]^{T},
\end{aligned}
$$

we have $P=[a, b]^{T}=\left(B^{T} B\right)^{-1} B^{T} y_{N}$.

The whitening response result of the grey model and the prediction model of the original series $\ddot{\mathscr{O}}^{(0)}$ can be obtained by regressive operations.

$$
\hat{x}^{(1)}(k+1)=\left(x^{(0)}(1)-\frac{b}{a}\right) \mathrm{e}^{-a k}+\frac{b}{a}, k=0,1,2, \cdots, n
$$

The reference values of accuracy indicators based on the grey model such as relative error, ratio of standard deviation and small error probability are listed in Table $\mathbf{1 .}$

When the test grade is above $2^{\text {nd }}$, we can apply this model directly. When the test grade is below $2^{\text {nd }}$, this model cannot be used to make predictions, unless some modifications are applied to the model to pass the test.

\subsection{Combined Model}

Based on the exponential smoothing method and the grey model, we can establish the combined model as follows:

$$
\left\{\begin{array}{l}
\hat{x}_{t}=\sum_{i=1}^{2} w_{i}\left(f_{t i}+\alpha \varepsilon_{t i}\right) \quad t=1,2, \cdots, n \text { and } 0 \leq \alpha \leq 1 \\
\sum_{i=1}^{2} w_{i}=1
\end{array}\right.
$$

Where $w_{1}$ and $w_{2}$ are the weight values, and $f_{t 1}, f_{t 2}$ and $\ddot{D}_{t}$ denote the predictive values of the exponential smoothing 
Table 2. Spare parts consumption amount from 2010 to 2014.

\begin{tabular}{|c|c|c|c|c|c|}
\hline $\begin{array}{c}\text { Spare Parts } \\
\text { Consumption Amount }\end{array}$ & 98 & 100 & 104 & 102 & 104 \\
\hline
\end{tabular}

Table 3. Comprehensive comparison of three prediction models.

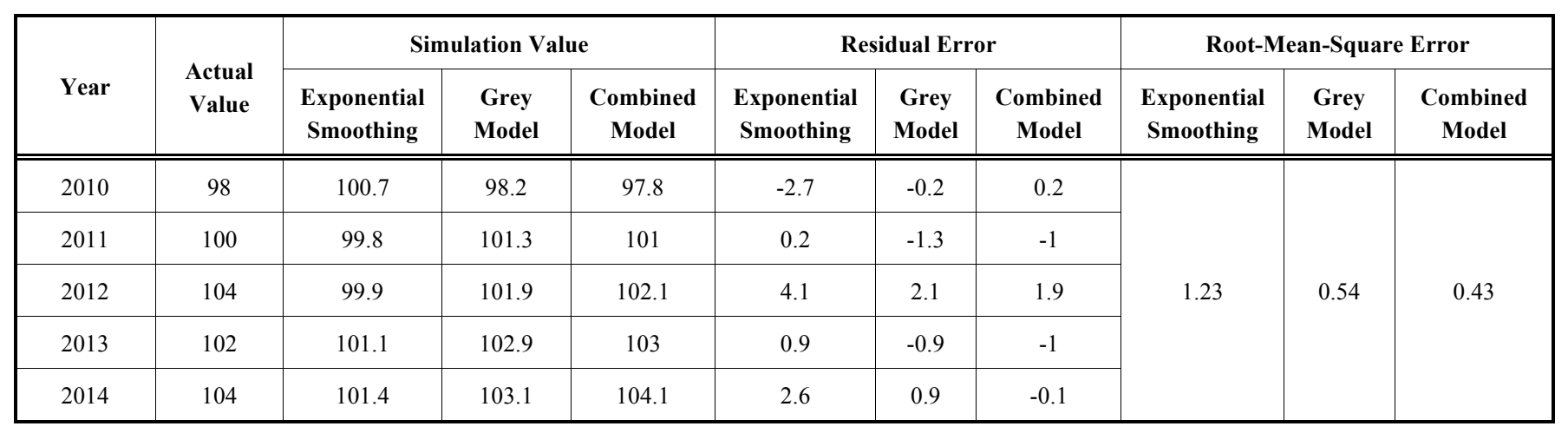

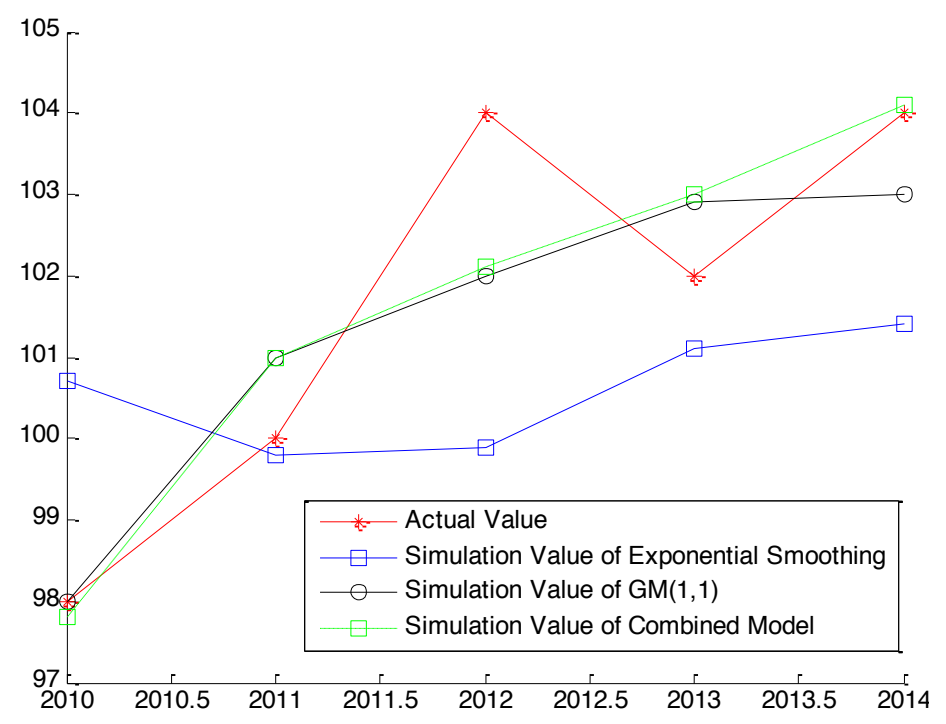

Fig. (1). Simulation values of three prediction models.

method, the grey model and the combined model, respectively. It was assumed that the value of the predictive error was around a fixed value, and $\alpha \sum_{i=1}^{m} w_{i} \varepsilon_{t i}$ can be denoted as the constant $a$, then $\hat{x}_{t}=a+\sum_{i=1}^{2} w_{i} f_{t i}, t=1,2, \mathrm{~L}, n$. Actually, $a$ is the weighted average value of each single prediction model.

\section{APPLICATION}

Given the consumption data of a specific kind of spare parts in 2010-2014, the detailed data list is shown in Table 2. Assuming that the amount of this kind of equipment is constant, the amount of spare parts consumption of this equipment in 2015 is predicted.
(1) By analyzing Table 2, we can find that the spare parts consumption data showed random changes in the variable around a fixed value, thus the single exponential smoothing method to was adopted for prediction. The value of smoothing coefficient $\alpha$ influenced the predictions. The average relative error reached the minimum when $\alpha=0.3$, which was obtained by verification tests. Therefore, the spare parts consumption prediction model became:

$$
\hat{x}_{t+1}=S_{t}^{(1)}=0.3 x_{t}+0.7 \hat{x}_{t} \quad(t \geq 1)
$$

(2) According to the historical consumption data of spare parts listed in Table $\mathbf{2}$, the grey model can be established as shown below:

$$
\hat{x}^{(1)}(k+1)=10363 \mathrm{e}^{0.0097 k}-10265, k \geq 0
$$


After regression operation, the simulation values of original series were as follows:

$$
\hat{x}^{(0)}=\left\{\hat{x}^{(0)}(1), \hat{x}^{(0)}(2), \cdots, \hat{x}^{(0)}(5)\right\}=\{98,101,102,102.9,103\} .
$$

The accuracy indicators of the grey model were $\Delta=0.0078<0.01, C=0.4681<0.5$, and $p=0.8$. Therefore, the precision of this model was of $2^{\text {nd }}$ grade, which can be used to predict the next year's spare parts consumption.

(3) After combining the exponential smoothing method with the grey model, the weight coefficient of the exponential smoothing method $w_{1}=-0.07$ was obtained along with the weight coefficient of the grey model $w_{2}=1.07$ and the constant $a=-0.03$.

The simulation values of three prediction models are shown in Fig. (1), and the comparative analysis of each predictive model is listed in Table $\mathbf{3}$.

From Table 3, it is obvious that the root-mean-square error of the combined model was the minimum error. Therefore, the combined prediction model can enhance the predictive precision, and the predictive value of this kind of spare parts was 106 in 2014.

\section{CONCLUSION}

The predictive precision of the combined model applied to predict the short-term consumption of spare parts can effectively be enhanced by using the information of the expo- nential smoothing method and the grey model synthetically. The application of this combined model can be extended for long-term prediction, and other single predictive models can be combined aimed at solving different problems. Moreover, this combined model can also be utilized in other predictive fields.

\section{CONFLICT OF INTEREST}

The authors confirm that this article content has no conflict of interest.

\section{ACKNOWLEDGEMENTS}

Declared none.

\section{REFERENCES}

[1] J. Zhao, T. Xu, Y. Liu, and Y. Yin, "Consumption forecasting of missile spare parts based on rough set, entropy weight and improved SVM", Acta Armamentarii, vol. 33, pp. 1258-1265, 2012.

[2] X. Ni, H. Zuo, F. T. Chen, and X. Rong, "Civil aircraft rotable spare parts forecasting", Journal of Nanjing University of Aeronautics \& Astronautics, vol. 41, pp. 253-256, 2009.

[3] D. Li, Z. Zhang, and T. Liu, "A regulated method of initial spare based on the Bayes method", Systems Engineering-Theory \& Practice, vol. 33, pp. 2967-2971, 2013.

[4] D. Xu, "A further study on the exponential smoothing estimation method for parameters of forecasting model and its application". Systems Engineering-Theory \& Practice, vol. 2, pp. 25-30, 2013.

[5] S. Li, and K. Liu, "Optimized dynamic exponential smoothing model and its applications", Journal of Systems Engineering, vol. 18, pp.163-167, 2003.

[6] H. Mu, W. Wang, Y. Ning, and G. Li, "Study of Energy Consumption Prediction Based on Improved Grey Model", Journal of Dalian University of Technology, vol. 51, pp.493-497, 2011.

(C) Shenyang et al.; Licensee Bentham Open.

This is an open access article licensed under the terms of the Creative Commons Attribution Non-Commercial License (http://creativecommons.org/licenses/by-nc/4.0/) which permits unrestricted, non-commercial use, distribution and reproduction in any medium, provided the work is properly cited. 